\title{
Centralized MIMO Radar MFSK Waveform Based on Frequency Division Multiplexing
}

\author{
Wei Wang \\ Shenyang Institute of Automation, \\ Chinese Academy of Sciences, \\ Shenyang 110016, China, \\ wangwei2@sia.cn, Corresponding \\ authorInstitutes for Robotics and \\ Intelligent Manufacturing, Chinese \\ Academy of Sciences, Shenyang \\ 110169, China.University of Chinese \\ Academy of Sciences, Beijing 100049, \\ China.Key Laboratory on Intelligent \\ Detection and Equipment Technology \\ of Liaoning Province, Shenyang \\ 100179, China.
}

\author{
Jinsong Du \\ Shenyang Institute of Automation, \\ Chinese Academy of Sciences, \\ Shenyang 110016, China.Institutes for \\ Robotics and Intelligent \\ Manufacturing, Chinese Academy of \\ Sciences, Shenyang 110169 , \\ China.Key Laboratory on Intelligent \\ Detection and Equipment Technology \\ of Liaoning Province, Shenyang \\ 100179, China.
}

\author{
Jie Gao \\ Shenyang Institute of Automation, \\ Chinese Academy of Sciences, \\ Shenyang 110016, China.Institutes for \\ Robotics and Intelligent \\ Manufacturing, Chinese Academy of \\ Sciences, Shenyang 110169, \\ China.Key Laboratory on Intelligent \\ Detection and Equipment Technology \\ of Liaoning Province, Shenyang \\ 100179, China.
}

\begin{abstract}
The centralized MIMO continuous wave radar will play an important role in in vehicle assisted driving/autonomous driving. MIMO radar waveform needs to meet orthogonality to ensure that the signals can be separated. This paper proposes a centralized MIMO radar LFM waveform, and further proposes a centralized MIMO Radar MFSK waveform based on frequency division multiplexing. The simulation results confirm the validity of the waveform.
\end{abstract}

\section{CCS CONCEPTS}

- Computing methodologies $\rightarrow$ Modeling and simulation; Model development and analysis.

\section{KEYWORDS}

MIMO radar, LFM, MFSK, frequency division multiplexing

\section{ACM Reference Format:}

Wei Wang, Jinsong Du, and Jie Gao. 2021. Centralized MIMO Radar MFSK Waveform Based on Frequency Division Multiplexing. In 2021 2nd International Conference on Artificial Intelligence and Information Systems (ICAIIS '21), May 28-30, 2021, Chongqing, China. ACM, New York, NY, USA, 5 pages. https://doi.org/10.1145/3469213.3470405

\section{INTRODUCTION}

MIMO radar $[1,2]$ was proposed under the inspiration of MIMO theory and space-time coding theory applied to communication systems and made major breakthroughs. Bliss and Forsythe studied the degree of freedom and spatial resolution of a centralized MIMO

Permission to make digital or hard copies of all or part of this work for personal or classroom use is granted without fee provided that copies are not made or distributed for profit or commercial advantage and that copies bear this notice and the full citation on the first page. Copyrights for components of this work owned by others than ACM must be honored. Abstracting with credit is permitted. To copy otherwise, or republish, to post on servers or to redistribute to lists, requires prior specific permission and/or a fee. Request permissions from permissions@acm.org.

ICAIIS '21, May 28-30, 2021, Chongqing, China

(c) 2021 Association for Computing Machinery.

ACM ISBN 978-1-4503-9020-0/21/05 . .\$15.00

https://doi.org/10.1145/3469213.3470405 radar [3, 4]. In the face of complex working environments and detection scenarios, the centralized MIMO radar adopts flexible design of the transmit waveform to improve the overall performance of the radar system[5]. The above results prove that the centralized MIMO continuous wave radar will have broad prospects in vehicle assisted driving/autonomous driving technology.

The centralized MIMO continuous wave radar for vehicle assisted driving/autonomous driving applications needs to meet certain constraints. The primary criterion for the waveform design of the centralized MIMO continuous wave radar is to ensure the separation and reception of signals. Time division multiplexing and frequency division multiplexing are the most direct methods to solve the above problems. Rohling $\mathrm{H}$ et al. studied typical continuous wave radar waveforms such as MFSK signals and FM sequence signals, and proposed a continuous wave MIMO radar MFSK waveform based on time division multiplexing and a continuous wave MIMO radar waveform based on frequency division multiplexing.

Based on the time-division multiplexed continuous wave MIMO radar MFSK waveform[6], each transmitting antenna has a separate time-occupied radar channel, and each antenna has a separate duration of $T$. Then the sampling frequency of the echo signal of each receiving antenna is $1 / T$, and the transmitted signal can be separated according to the order of occupied channels.

The continuous wave MIMO radar waveform based on frequency division multiplexing considers the situation of two transmitting array elements[7]. The transmission signals of the two transmitting array elements have the same frequency modulation slope, frequency modulation bandwidth and duration. The transmission signal of the second transmitting array element has a frequency shift $f_{\text {shift }}$, so the transmission signals of the two antennas occupy different frequencies at the same time. The frequency shift $f_{\text {shift }}$ should be greater than the maximum beat signal frequency $f_{B \text {, max }}$ to ensure that the receiving channel can process the two transmitted signals separately and save them as different two-dimensional data matrices. 


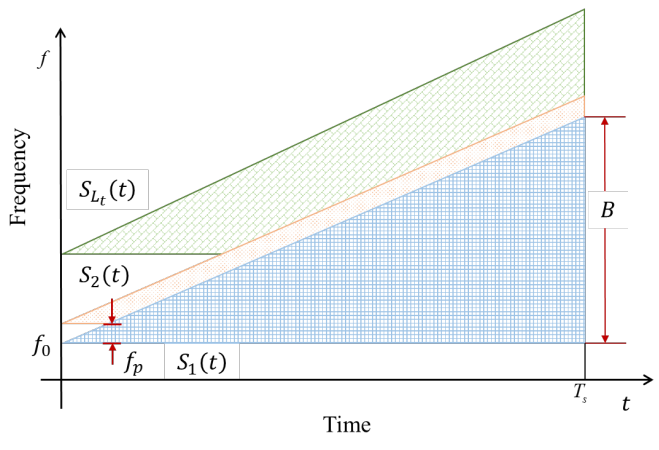

Figure 1: The centralized MIMO radar LFM waveform

The above two signal principles are relatively simple and provide ideas for MIMO radar waveform design. Based on this, this paper designs a centralized MIMO radar MFSK waveform based on frequency division multiplexing, and verifies the effectiveness of the waveform through simulation.

\section{CENTRALIZED MIMO RADAR LFM WAVEFORM}

The centralized MIMO radar system includes $L_{t}$ transmitting antennas and $L_{r}$ receiving antennas. Both the transmitting antennas and the receiving antennas are uniform linear arrays, in which the distance between the transmitting antennas is $d$ and the distance between the receiving antennas is $L_{t} d$. The MIMO radar system is equivalent to a virtual array composed of $L_{t} \times L_{r}$ antennas.

Assume that the reference signal of the first transmitting antenna is

$$
S_{1}(t)=p(t) \cos \left\{2 \pi\left[f_{0}+\frac{1}{2} \mu t\right] t\right\}
$$

Where, $p(t)$ is the signal envelope, $f_{0}$ is the signal carrier frequency, $\mu=B / T_{s}$ is the frequency modulation slope, $B$ is the signal bandwidth, $T_{s}$ is the duration.

The signal of the $m$-th transmitting antenna is

$$
S_{m}(t)=p(t) \cos \left\{2 \pi\left[f_{0}+(m-1) f_{p}+\frac{1}{2} \mu t\right] t\right\}
$$

Assume that $\tau_{\max }$ represents the round-trip time of the electromagnetic wave propagation of the farthest target detected by the radar, $f_{p}$ represents the frequency step of the transmitted signal of the adjacent transmitting antenna and $f_{p}>\tau_{\max }[7]$. The waveform is shown in Figure 1

The transmitted signal is reflected by $K$ targets in the detection space, and the echo signal of the $n$-th receiving antenna is:

$$
R_{n}(t)=\sum_{k=1}^{K} \sum_{m=1}^{L_{t}} G_{n}\left(t, m, \tau_{m, k, n}\right) S r_{n}\left(t, m, \tau_{m, k, n}\right)
$$

Where

$$
\begin{aligned}
& S_{n}\left(t, m, \tau_{m, k, n}\right)= \\
& \exp \left\{j 2 \pi\left[f_{0}+(m-1) f_{p}+\frac{1}{2} \mu t_{m, k, n}+f_{D, k, m}\right] t_{m, k, n}\right\} \\
& G_{n}\left(t, m, \tau_{m, k, n}\right)=A_{k} p\left(t-\tau_{m, k, n}\right)
\end{aligned}
$$

Where, $G_{n}\left(t, m, \tau_{m, k, n}\right)$ represents echo signal envelope, $A_{k}$ represents the reflection coefficient of the $k$-th target in the detection space. $t_{m, k, n}=t-\tau_{m, k, n}, \tau_{m, k, n}$ represents the time delay for the signal of the $m$-th transmitting antenna to return to the $n$-th receiving antenna after being reflected by the $k$-th target. $f_{D, k, m}$ represents the Doppler shift of the transmitted signal of the $m$-th transmitting antenna due to the $k$-th target.

$$
f_{D, k, m}=2\left(f_{0}+(m-1) f_{p}\right) v_{k} / c
$$

Where, $v_{k}$ represents the velocity of the $k$-th target relative to the radar.

The echo signal of the $n$-th receiving antenna is mixed with the reference signal, then the beat signal of the $n$-th receiving antenna is

$$
B_{n}(t)=\sum_{k=1}^{K} \sum_{m=1}^{L_{t}} G_{B}\left(t, m, \tau_{m, k, n}\right) S_{B}\left(t, m, \tau_{m, k, n}\right)
$$

Where

$$
S_{B n}\left(t, m, \tau_{m, k, n}\right)=\exp \left\{j 2 \pi \cdot f_{B, k, m, n} t+\varphi_{m, k, n}\right\}
$$

Where, $G_{B}\left(t, m, \tau_{m, k, n}\right) \quad$ represents signal envelope, $f_{B, k, m, n}$ represents the beat frequency for the signal of the $m$-th transmitting antenna to return to the $n$-th receiving antenna after being reflected by the $k$-th target.

$$
f_{B, k, m, n}=(m-1) f_{p}-\mu \tau_{m, k, n}+f_{D, k, m}
$$

In formula (9), $(m-1) f_{p}$ is the frequency shift caused by the variable carrier frequency, and $-\mu \tau_{m, k, n}$ is the frequency shift caused by the target distance.

The beat signal can be divided into $L_{t}$ frequency bands, and the signal is subjected to multi-channel filtering processing to form $L_{t}$ channels for each transmitting antenna.

$$
\begin{aligned}
& D_{n, m}(t)= \\
& \sum_{k=1}^{K} G_{B S} n\left(t, m, \tau_{m, k, n}\right) \exp \left\{j 2 \pi\left(f_{k, m, n} t+\varphi_{m, k, n}\right)\right\}
\end{aligned}
$$

The sampling frequency of each channel is $f_{s}$, and the signal passes through a digital low-pass filter, and the signal spectrum is obtained through FFT processing. The $K$ spectral lines in the frequency spectrum correspond to $K$ targets in the detection space. $f_{k, m, n}$ is the frequency of the $k$-th target corresponding to the signal of the $n$-th receiving antenna received by the $m$-th channel.

$$
f_{k, m, n}=f_{B, k, m, n}+f_{D, k, m}
$$

Where, $f_{B, k, m, n}$ and $f_{D, k, m}$ correspond to the distance and speed information of the target. The Doppler frequency $f_{D, k, m}$ can be extracted from the frequency spectrum in the slow time domain by multi-period signals.

The multi-carrier frequency modulation continuous wave centralized MIMO radar system architecture is shown in Figure 2

\section{CENTRALIZED MIMO RADAR MFSK WAVEFORM}

Radar waveform generation is based on the combination of Phase Locked Loop (PLL) and Voltage Controlled Oscillator (VCO), and each transmission link is controlled by the frequency control word 




Figure 2: The centralized MIMO radar system

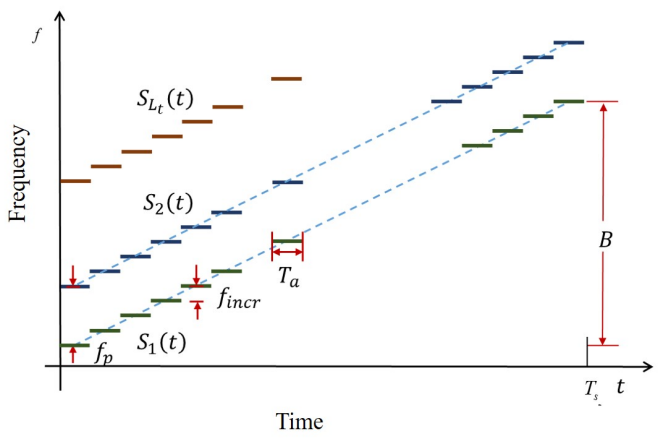

Figure 3: The Centralized MIMO radar MFSK waveform

to control the parameters of the transmission signal to generate a stepped frequency chirp signal.

The transmitted signal of each antenna realizes the multi-carrier frequency centralized MIMO radar waveform according to the MFSK signal mode. The frequency step of the transmitted signal of the adjacent transmitting antenna is $f_{p}$. The duration of each signal at a certain frequency is $T_{a}$. The frequency step of the MFSK signal is $f_{\text {incr }}$. The number of steps is $N-1$, the frequency modulation bandwidth is $B=(N-1) f_{\text {incr }}$, and the frequency modulation duration is $T_{s}=N T_{a}$. The received signals are mixed to the baseband, and the beat signals are discretely sampled. The sampling frequency is $1 / T_{a}$. The centralized MIMO radar MFSK waveform is shown in Figure 3

MFSK signal maintains the characteristics of LFM signal. Formula (7) and (8) show that the beat signal frequency is mainly related to the signal bandwidth $B$ and the frequency modulation duration $T_{s}$.

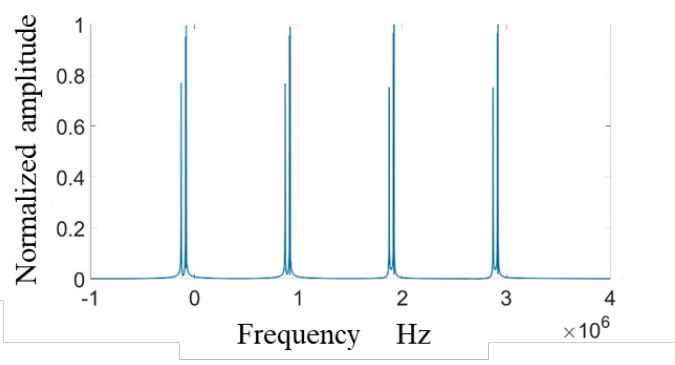

Figure 4: The beat signal spectrum of the first antenna

$f_{\text {incr }}$ and $T_{a}$ are also key parameters that affect signal performance. Different frequency components in the step frequency make the spectrum of the beat signal more complicated.

\section{SIMULATIONS}

Assuming that the centralized MIMO radar system has 4 transmitting antennas and 4 receiving antennas. Both the transmitting antennas and the receiving antennas are uniform linear arrays. Set the reference signal carrier frequency $f_{0}$ to $24.000 \mathrm{GHz}$, bandwidth Bto $200 \mathrm{MHz}$, and frequency modulation duration $T_{s}$ to $1 \mathrm{~ms}$. The maximum detection distance of the radar is $180 \mathrm{~m}$, and the frequency step of the transmitted signal of the adjacent transmitting antenna $f_{p}$ should be greater than $0.48 \mathrm{MHz}$, which is set to $1 \mathrm{MHz}$. The main parameters are shown in TABLE 1 .

Assuming that there are 3 targets in the detection space, which are used to verify the effect of the centralized MIMO radar LFM waveform. The target parameters are shown in TABLE 2.

The echo signal of the receiving antenna is mixed with the reference signal. The beat signal of the first receiving antenna can be divided into 4 frequency bands, as shown in Figure 4. The frequency interval between each subband is equal to the frequency step of the frequency step of the transmitted signal of the adjacent transmitting antenna, and each subband includes 3 frequency peaks, corresponding to 3 targets in the detection space. In addition to being divided into multiple subbands due to frequency stepping, the frequency spectrum is mainly affected by the frequency shift caused by the target distance and the Doppler frequency caused by the target velocity.

The beat signal passes through the filters of 4 channels respectively. AD sampling and digital low-pass filtering are performed on the band-pass filtered signal. The sampling frequency is $1 \mathrm{MHz}$, and the frequency spectrums of each subband signals after filtering are moved to the baseband. The signal separation effect is shown in Figure 5

The above simulation results confirm the effectiveness of the centralized MIMO radar LFM waveform and its signal separation method. It is assumed that MFSK waveform parameters of centralized MIMO radar are the same as LFM waveform parameters. The two parameters of the frequency step of the MFSK signal $f_{i n c r}$ and the duration of each step frequency $T_{a}$ are added, as shown in TABLE 3.

Assuming that there are 3 targets in the detection space. The parameters are the same as the simulation parameters in TABLE 2. 
Table 1: The Main parameters of the centralized MIMO Radar LFM waveform able Styles

\begin{tabular}{ll}
\hline The Parameters & The Symbol \\
\hline The reference signal carrier frequency & $f_{0}=24.000 \mathrm{GHz}$ \\
The bandwidth & $B=200 \mathrm{MHz}$ \\
The frequency modulation duration & $T_{s}=1 \mathrm{~ms}$ \\
The frequency step of the transmitted signal of the adjacent transmitting & $f_{p}=1 \mathrm{MHz}$ \\
antenna & \\
The sampling frequency & $f_{s}=1 \mathrm{MHz}$ \\
The number of transmitting antennas & $L_{t}=4$ \\
The number of receiving antennas & $L_{r}=4$ \\
\hline
\end{tabular}

Table 2: The Target parameters

\begin{tabular}{lll}
\hline No. & Distance $(\mathbf{m})$ & Velocity $(\mathbf{m} / \mathbf{s})$ \\
\hline 1 & 60 & -5 \\
2 & 65 & 0 \\
3 & 100 & 30 \\
\hline
\end{tabular}

Table 3: The added parameters of the MIMO radar MFSK signal

\begin{tabular}{ll}
\hline The Parameters & The Symbol \\
\hline The frequency step of the MFSK signal & $f_{\text {incr }}=200 \mathrm{kHz}$ \\
The duration of each step frequency & $T_{a}=1 \times 10^{-3} \mathrm{~ms}$ \\
\hline
\end{tabular}



Figure 5: The signal separation effect of LFM signal

The beat signal of the first receiving antenna can be divided into 4 frequency bands, as shown in Figure 6. The frequency interval between each subband is equal to the frequency step of the frequency step of the transmitted signal of the adjacent transmitting antenna, and each subband includes the expected 3 frequency peaks, and there are also 3 frequency peaks introduced by the aliasing of the frequency step of the MFSK signal $f_{\text {incr }}$, which can be filtered out by a band-pass filter.

The subsequent processing method is the same as the LFM waveform. The signal separation effect is shown in Figure 7

The comparison effect of the centralized MIMO radar LFM waveform and the centralized MIMO radar MFSK waveform is shown in Figure 8 . The simulation results confirm that the MFSK waveform has similar effects.

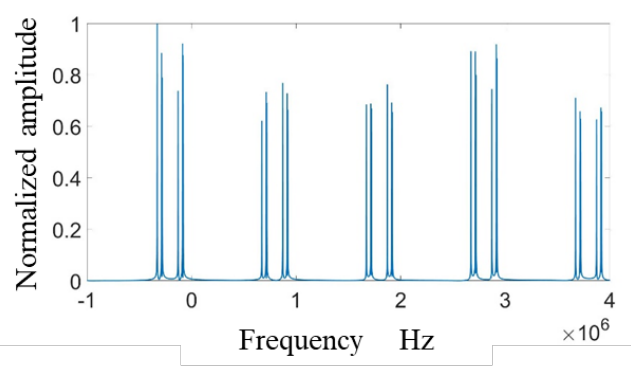

Figure 6: The beat signal spectrum of the first antenna

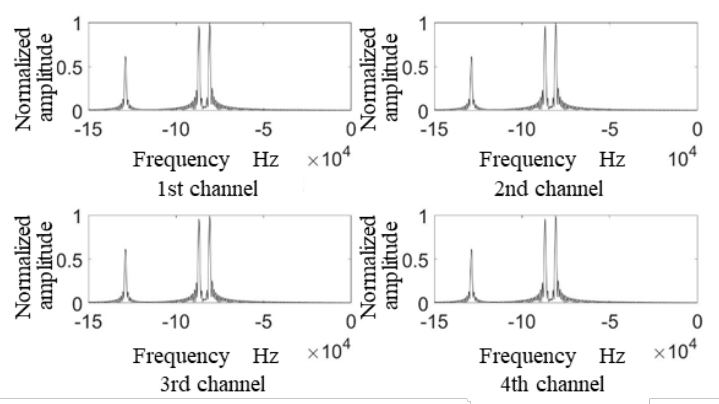

Figure 7: The signal separation effect of MFSK signal 




The first channel separated from the first receiving antenna

Figure 8: LFM waveform and MFSK waveform effect comparison

\section{CONCLUSION}

This paper proposes a centralized MIMO radar LFM waveform, and further proposes a centralized MIMO Radar MFSK waveform based on frequency division multiplexing. The simulation results confirm the validity of the waveform. The above work establishes the foundation for further research on MIMO radar OFDM waveforms.

\section{ACKNOWLEDGMENTS}

This work was supported by the National Natural Science Foundation of China (Grant No.61703393), by the Natural Science Foundation of Liaoning Province (No. 2019-MS-343, No. 20180520016 and No. 20180520008) and by the K.C.Wong Education Foundation.

\section{REFERENCES}

[1] Fishler E, Haimovich A, Blum R, et al. MIMO radar: an idea whose time has come[C]. Radar Conference, 2004. Proceedings of the IEEE. IEEE Xplore, 2004:71-78.

[2] J Li , P Stoica. MIMO Radar Signal Processing[M] . New York :Wiley, 2009 . 1-10.

[3] Bliss D W, Forsythe K W. Multiple-input multiple-output (MIMO) radar and imaging: degrees of freedom and resolution[C]. Signals, Systems and Computers, 2004. Conference Record of the Thirty-Seventh Asilomar Conference on. IEEE, 2003:5459 Vol.1.

[4] Forsythe K W, Bliss D W, Fawcett G S. Multiple-input multiple-output (MIMO) radar: performance issues[C]. Signals, Systems and Computers, 2004. Conference Record of the Thirty-Eighth Asilomar Conference on. IEEE, 2005:310-315 Vol.1.

[5] Friedlander B. Waveform Design for MIMO Radars[J]. Aerospace \& Electronic Systems IEEE Transactions on, 2007, 43(3):1227-1238.

[6] Zwanetski A, Rohling $\mathrm{H}$. Continuous wave MIMO radar based on time division multiplexing[C]. Radar Symposium. IEEE, 2012:119-121.

[7] Zwanetski A, Kronauge M, Rohling H. Waveform design for FMCW MIMO radar based on frequency division[C]. Radar Symposium. IEEE, 2013:89-94 